\title{
Overview of Pulmonary Physiotherapy in Symptoms and Complication of COVID 19
}

\author{
Heeral Joshi ${ }^{1}$, Shweta Sharma1, Pooja Anand ${ }^{2}$ \\ ${ }^{1}$ Assistant Professor, Department of Physiotherapy Manav Rachna International Institute of Research and Studies, \\ Faridabad, Haryana, ${ }^{2}$ Professor/H.O.D, Physiotherapy Manav Rachna International Institute of Research and \\ Studies, Faridabad, Haryana
}

\begin{abstract}
COVID 19 or CORONA as we know it has not only become a global pandemic but a real threat to humanity which is proving itself as a third world war for the world. Everyone is talking about the basic preventive measures for fighting with this situation let it be proper hygiene, social distancing or allied healthcare measures. The basic symptoms of the covid-19 patients, the virus attacks healthy as well as immune-compromised individuals. The onset of virus starts from inhalation of droplets impregnated with virus which shows its effect on larynx and via it, progresses to lungs covering the respiratory system causing acute respiratory infections. Once in lungs it makes it as a strong hold on ground for further deterioration of immunity and respiratory functions which leads to fatal conditions like pneumonia acute respiratory distress syndromes. Now if by various respiratory physiotherapeutic practices if we can increase the strength of lungs it can actually increase the recovery of the patients with the aim of maintaining the bronchial hygiene and strengthening the respiratory muscles at various stages. Bronchial toileting, positioning can be helpful in dealing with complications like pneumonia and ARDS, where pursed lip breathing and ACBT can be helpful in dealing with breathlessness in early stages.
\end{abstract}

Keywords: COVID 19, Pneumonia, ARDS, Bronchial toileting, Positioning, Pursed lip Breathing, ACBT.

\section{Introduction}

The recent COVID -19 outbreaks has been declared as the global health emergency, India till date has already registered 909 covid-19 positive cases with 19 deaths and 40 cured cases ${ }^{(1)}$. Indian government has taken various preventive measures against the virus and has taken a major step by locking down the whole country. Still we are getting various positive cases of covid-19 ${ }^{(1)}$. India is at a brink of entering at tertiary level. Various preventive measures for the people in India and intervention are getting implemented with the cases who are positive COVID 19. Since the complications of COVID 19 affects directly the respiratory system it has become important that the few strong measures should be highlighted for prevention and rehabilitation.

\section{Method}

Various international research articles have been reviewed for this study so that a standard protocol for the intervention of respiratory care should be inoculated as well as to establish the importance of respiratory therapy as the preventive measure

Findings: The incubation period of covid-19 is from 1 day to $14^{\text {th }}$ day the symptoms which are initially seen are fever, sore throat, dry cough, breathlessness and headache. The cases which have been resolved spontaneously are the one who were not having any secondary issues like diabetes, hypertension, any renal diseases, or who were not immune-suppressed ${ }^{(3)}$. However rest of them who are undergoing fatal complications like organ failure, septic shock, pneumonia \& acute respiratory distress syndrome. Notably people who intensive care, are with multiple co morbidities including cerebro-vascular diseases, cardiovascular diseases and endocrine disease, digestive and respiratory disease ${ }^{(4.5 .6)}$. More symptoms which were reported from ICU are severe dyspnoea, dizziness, anorexia and abdominal pain ${ }^{(4.5 .6)}$.

Few doctors from the city Jaipur, Rajasthan has 
treated the patients with drugs used in HIV and they found it to be useful as no effective anti-viral treatment for covid-19 is available or researched till now. Whole community of doctors and pharmacist are rigorously researching for antidote of covid-19. On a temporary note the doctors are treating patients symptomatically via anti pyretic therapy, cough expectorants for non productive cough. Broad spectrum antibiotics are used in cases diagnosed with sepsis post covid-19 within an hour. Patients are also reporting with various fungal $\&$ bacterial infections are also treated with the same protocol.

According to World Health Organization acute respiratory syndrome is the first sign along with temperature $=>38$ degree Celsius ${ }^{(1)}$. Preventive measures which taken are social distancing and regular hand hygiene where nobody is talking about bronchial hygiene. By maintaining bronchial hygiene we can save the patient from being collapsing ${ }^{(7)}$.

Preventive measures in medical setups are nearest distance is 2 meters for 15 minutes as such patients are recorded with the temperature of more than 38 degree Celsius. No passive bronchial toileting can be indicated in acute respiratory infection or acute stages of patients with covid-19 pursed lip breathing (where the patient is asked to purse his lips and to breathe in via nose and exhale with the pursed lips), positioning and relaxation technique can help patient with symptoms of shortness of breath ${ }^{(7,9)}$.

The Active Cycle of Breathing Technique consisted of 4-6 breathing control breaths, 3-4 thoracic expansion exercises and the forced expiration technique including 4-6 breathing control breaths combined with 2-3 huffs followed by cough ${ }^{(11)}$. It can also be used as a respiratory therapy if the patient is in condition to perform as it has proved as a great measure to deal with respiratory failure $^{(10)}$, it can be a preventive measure for further stages of covid-19.

Where as in pneumonia along with covid-19 in acute stages bronchial drainage is contra indicated, the patient being ill \& lung being totally consolidated and unproductive ${ }^{(7)}$. Breathing exercises to maintain maximum ventilation in all areas of lung plus foot exercises (ankle pump) can be useful ${ }^{(12)}$. Once entered the hepatization stage physiotherapy is to aid in cleaning the exudates is required ${ }^{(7)}$.

In cases with ARDS of covid-19 frequent changes of positioning must be used in order to bring improvement in ventilation/perfusion ratio in certain parts of lungs and to prevent prolong dependency on any one section of lung ${ }^{(7)}$. This improvement is only temporary but often leads to temporary improvement in oxygenation ${ }^{(7)}$. Few study also have documented that prone positions significantly increase oxygenation and decrease driving pressure in patients with $\operatorname{ARDS}^{(13)}$

In general people can use various breathing exercise which can help in maintaining the bronchial hygiene and strength of respiratory system on a daily basis which will be a preventive measure on a larger note ${ }^{(8)}$.

\section{Conclusion}

In such unprecedented situations which the whole world is facing, we as a developing country not only have to work on our primary health care system but simultaneously we have to develop the second line of health defense measures so that we not only reduces the risk of primary infections but also strengthen the respiratory system to fight this virus effectively in case one get infected be it a healthy individual or a immunecompromised one.

\section{Conflict of Interest: No}

Source of Funding: Self as no such major fund is involved

Ethical Clearance: As it is a review so no ethical clearance required

\section{References}

1. World Health Organization. Coronavirus disease 2019 ( COVID-19): situation report, 51.

2. Xu Z, Shi L, Wang Y, Zhang J, Huang L, Zhang C, Liu S, Zhao P, Liu H, Zhu L, Tai Y. Pathological findings of COVID-19 associated with acute respiratory distress syndrome. The Lancet respiratory medicine. 2020 Feb 18.

3. Bai Y, Yao L, Wei T, Tian F, Jin DY, Chen L, Wang M. Presumed asymptomatic carrier transmission of COVID-19. Jama. 2020 Feb 21.

4. Novel CP. The epidemiological characteristics of an outbreak of 2019 novel coronavirus diseases (COVID-19) in China. Zhonghua liu xing bing xue za zhi= Zhonghua liuxingbingxue zazhi. $2020 \mathrm{Feb}$ 17;41(2):145.

5. Gao J, Tian Z, Yang X. Breakthrough: Chloroquine 
phosphate has shown apparent efficacy in treatment of COVID-19 associated pneumonia in clinical studies. Bioscience trends. 2020.

6. Pan F, Ye T, Sun P, Gui S, Liang B, Li L, Zheng D, Wang J, Hesketh RL, Yang L, Zheng C. Time course of lung changes on chest CT during recovery from 2019 novel coronavirus (COVID-19) pneumonia. Radiology. 2020 Feb 13:200370.

7. Parker AM, Sricharoenchai T, Needham DM. Early rehabilitation in the intensive care unit: Preventing impairment of physical and mental health. Current physical medicine and rehabilitation reports. 2013 Dec 1;1(4):307-14.

8. Ries AL, Bauldoff GS, Carlin BW, Casaburi R, Emery CF, Mahler DA, Make B, Rochester CL, ZuWallack R, Herrerias C. Pulmonary rehabilitation: joint ACCP/AACVPR evidencebased clinical practice guidelines. Chest. 2007 May 1;131(5):4S-2S.

9. Sassi-Dambron DE, Eakin EG, Ries AL, Kaplan RM. Treatment of dyspnea in COPD: a controlled clinical trial of dyspnea management strategies. Chest. 1995 Mar 1;107(3):724-9.

10. Inal-Ince D, Savci S, Topeli A, Arikan H. Active cycle of breathing techniques in non-invasive ventilation for acute hypercapnic respiratory failure. Australian Journal of Physiotherapy. 2004 Jan 1;50:67-76.

11. Lewis LK, Williams MT, Olds TS. The active cycle of breathing technique: A systematic review and meta-analysis. Respiratory medicine. $2012 \mathrm{Feb}$ 1;106(2):155-72.

12. Niederman MS. Community-acquired pneumonia. Texbook of Critical Care. 2005:647-1.

13. Guerin C, Beuret P, Constantin JM, Bellani G, Garcia-Olivares P, Roca O, Meertens JH, Maia PA, Becher T, Peterson J, Larsson A. A prospective international observational prevalence study on prone positioning of ARDS patients: the APRONET (ARDS Prone Position Network) study. Intensive care medicine. 2018 Jan 1;44(1):22-37. 